\title{
DWDM-TO-OTDM Conversion by Time-Domain Optical Fourier Transformation
}

Mulvad, Hans Christian Hansen; Hu, Hao; Galili, Michael; Ji, Hua; Palushani, Evarist; Clausen, Anders; Oxenløwe, Leif Katsuo; Jeppesen, Palle

\section{Published in:}

Proceedings of the European Conference on Optical Communication (ECOC) 2011

Publication date:

2011

Document Version

Publisher's PDF, also known as Version of record

Link back to DTU Orbit

Citation $(A P A)$ :

Mulvad, H. C. H., Hu, H., Galili, M., Ji, H., Palushani, E., Clausen, A., Oxenløwe, L. K., \& Jeppesen, P. (2011). DWDM-TO-OTDM Conversion by Time-Domain Optical Fourier Transformation. In Proceedings of the European Conference on Optical Communication (ECOC) 2011 (pp. Mo.1.A.5). Optical Society of America. http://www.ecoc2011.org/

\section{General rights}

Copyright and moral rights for the publications made accessible in the public portal are retained by the authors and/or other copyright owners and it is a condition of accessing publications that users recognise and abide by the legal requirements associated with these rights.

- Users may download and print one copy of any publication from the public portal for the purpose of private study or research.

- You may not further distribute the material or use it for any profit-making activity or commercial gain

- You may freely distribute the URL identifying the publication in the public portal 


\title{
DWDM-to-OTDM Conversion by Time-Domain Optical Fourier Transformation
}

\author{
H.C. Hansen Mulvad, H. Hu, M. Galili, H. Ji, E. Palushani, A.T. Clausen, L.K. Oxenløwe, and P. Jeppesen \\ DTU Fotonik, Technical University of Denmark, Building 343, DK-2800 Kgs. Lyngby, Denmark \\ hchm@fotonik.dtu.dk
}

\begin{abstract}
We propose DWDM-OTDM conversion by time-domain optical Fourier transformation. Error-free conversion of a $16 \times 10 \mathrm{Gbit} / \mathrm{s} 50 \mathrm{GHz}$-spacing DWDM data signal to a $160 \mathrm{Gbit} / \mathrm{s}$ OTDM signal with a $2.1 \mathrm{~dB}$ average penalty is demonstrated.

OCIS codes: (060.4510) Optical communications; (190.4380) Nonlinear optics, four-wave mixing.
\end{abstract}

\section{Introduction}

Future high-capacity communication systems might require technologies for optical conversion between parallel and serial data formats [1]. Converting many low data rate parallel channels to fewer serial high data rate channels could simplify network management. A potential application is interfacing between lower speed access networks and core networks operating at high line rates. A DWDM-OTDM converter performs the parallel-to-serial conversion of dense wavelength division multiplexed (DWDM) low bit rate data channels into a single-wavelength optical timedivision multiplexed (OTDM) data channel at the aggregate bit rate. In this process, each DWDM channel must be confined within a separate OTDM time-slot, an operation which might become increasingly difficult for larger numbers of DWDM channels. Previous WDM-OTDM demonstrations are based on a variety of techniques with different degrees of complexity, see [1-2] and references therein. However, no publications have yet reported timedomain optical Fourier transformation (OFT) [3] for this functionality. The OFT technique inherently transfers the spectral profile of an optical waveform into the time-domain intensity profile (or vice-versa), and it is therefore a well-suited candidate for conversion between serial and parallel data formats [4].

Here, we propose to use time-domain OFT for DWDM-OTDM conversion, allowing large numbers of DWDM channels to be converted in a single switching operation. Time-alignment of the incoming WDM channels is necessary, but some tolerance towards misalignment can be expected from the time-lens effect associated with the OFT method [3,5]. The scheme is experimentally demonstrated on a $16 \times 10 \mathrm{Gbit} / \mathrm{s}$ DWDM signal with $50 \mathrm{GHz}$ channel spacing. The resulting $160 \mathrm{Gbit} / \mathrm{s}$ OTDM signal is demultiplexed with error-free performance, with an average penalty of only $2.1 \mathrm{~dB}$ compared to the DWDM channels.

\section{Principle}

Time-domain OFT of an optical waveform is achieved by a combination of parabolic phase-modulation (chirp rate $K$ ) and second order chromatic dispersion $D$. The principle is shown in the upper left inset in Fig. 1, where the input waveform is a DWDM data signal consisting of spectrally distinct channels (here synchronized in the time-domain). The OFT transfers the input spectral profile to the output temporal intensity profile, provided the condition $D=1 / K$ is fulfilled [3]. In this experiment, the phase-modulation is achieved by a four-wave mixing (FWM) process [5]. Linearly chirped, flat-top pulses of chirp rate $K / 2$ serve as pump signal (field $E_{p}(t)$ ), and the DWDM pulses act as probe signal (field $E_{s}(t)$ ). The flat-top pump pulses must fully overlap the DWDM pulses. The FWM process will then generate an idler field $E_{i}(t) \propto E_{p}^{2}(t) E_{s}^{*}(t)$, thus transferring the pump chirp onto the idler signal (with a factor 2). After the FWM, the idler is propagated through a dispersive fibre $D$ that cancels the FWM-generated chirp. The final result is a simultaneous temporal compression and time-interleaving of the DWDM channels into OTDM tributaries. A DWDM channel spacing $\Delta v$ is mapped into an OTDM tributary spacing $\Delta \tau$ according to $\Delta \tau=\Delta v / K$. The temporal shape of the output OTDM waveform is robust to timing misalignment of the incoming DWDM pulses, as long as the latter are overlapped by the pump. Within this tolerance, a timing shift at the OFT input will be translated into a spectral shift at the output [3]. However, this so-called time-lens effect is not experimentally verified in this work, where only the case of synchronous incoming DWDM channels is investigated.

\section{Experimental set-up}

The experimental set-up is shown in Fig. 1, including optical sampling oscilloscope (OSO) traces of the various waveforms (upper right insets). An erbium glass oscillator pulse-generating laser (ERGO-PGL) emits $10 \mathrm{GHz}$ pulses at a wavelength of $1542 \mathrm{~nm}$ with a temporal duration of $\sim 1.5 \mathrm{ps}$ full-width at half-maximum (FWHM). The ERGOPGL output pulses undergo self-phase modulation (SPM) in $400 \mathrm{~m}$ of dispersion-flattened highly non-linear fibre 


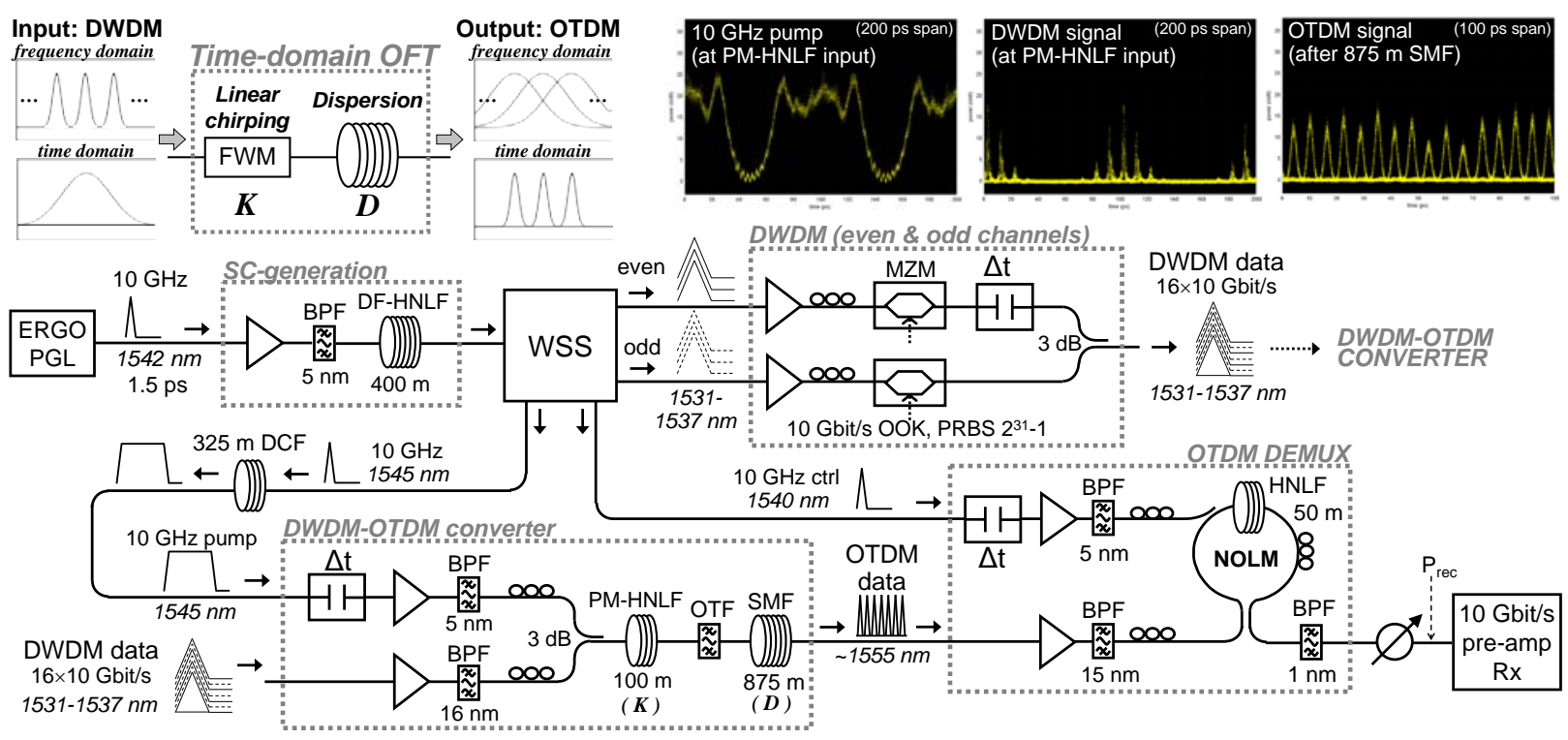

Fig. 1. Experimental set-up. Insets: Time-domain OFT principle (top left); waveforms measured with an OSO (top right).

(DF-HNLF) to generate a supercontinuum spanning the C-band, which is used as input to a programmable filter with 4 output ports (wavelength selective switch - WSS). The odd and even channels are obtained using two programmed filters consisting of eight $100 \mathrm{GHz}$-spaced Gaussian-profiled channels of spectral FWHM $13-14 \mathrm{GHz}$, both centered at $1534 \mathrm{~nm}$ with a relative shift of $50 \mathrm{GHz}$. The odd and even channels are directed to different WSS output ports, then separately encoded by on/off keying (OOK) with $10 \mathrm{Gbit} / \mathrm{s} 2^{31}-1$ PRBS patterns in Mach-Zehnder modulators (MZM), and finally combined using a 3-dB coupler, resulting in a single-polarisation 16×10 Gbit/s DWDM signal with $50 \mathrm{GHz}$ channel spacing. The DWDM-OTDM converter is optimised for a periodic $160 \mathrm{Gbit} / \mathrm{s}$ OTDM output with a tributary spacing of $\Delta \tau=6.25 \mathrm{ps}$, which requires $K=8 \mathrm{GHz} / \mathrm{ps}$. The pump pulses are obtained from the WSS using a $10^{\text {th }}$ order super-Gaussian filter of FWHM $300 \mathrm{GHz}$ at $1545 \mathrm{~nm}$, and then linearly chirped by dispersive propagation through a $325 \mathrm{~m}$ DCF, resulting in a broadened flat-top pulse of FWHM 75 ps and chirp rate $K / 2=4 \mathrm{GHz} / \mathrm{ps}$. The non-linear medium for the FWM between pump and data is a polarization-maintaining, elliptical core highly non-linear fiber (PM-HNLF) of length $100 \mathrm{~m}$, zero-dispersion wavelength $1545 \mathrm{~nm}$, dispersion slope $0.025 \mathrm{ps} /\left(\mathrm{nm}^{2} \cdot \mathrm{km}\right)$, and non-linear coefficient $\gamma \sim 10 \mathrm{~W}^{-1} \mathrm{~km}^{-1}$. The average input power to the PM-HNLF is $\sim 25 \mathrm{dBm}$ for the pump, and $\sim 5 \mathrm{dBm}$ for the DWDM signal. All DWDM pulses are synchronised and temporally aligned with the pump pulses at the PM-HNLF input using the variable time-delays $(\Delta t)$. After the FWM, the idler generated at $1555 \mathrm{~nm}$ is extracted using an optical tunable filter OTF (Santec OTF-350), and then passed through $875 \mathrm{~m}$ of standard SMF (with dispersion $D=1 / K$ ) to obtain the $160 \mathrm{Gbit} / \mathrm{s}$ OTDM signal. A non-linear optical loop mirror (NOLM) is used for time-demultiplexing the OTDM tributaries, using $10 \mathrm{GHz}$ Gaussian control pulses of $3.4 \mathrm{ps}$ FWHM at $1540 \mathrm{~nm}$ obtained from the WSS. The demultiplexed $10 \mathrm{Gbit} / \mathrm{s}$ tributaries are extracted by a $1 \mathrm{~nm}$ BPF, followed by bit-error rate (BER) measurement in a $10 \mathrm{Gbit} / \mathrm{s}$ pre-amplified receiver. For synchronisation of the receiver and PRBS generator, a $10 \mathrm{GHz}$ clock is extracted from the ERGO-PGL which is in free-running mode.

\section{Experimental results}

Fig. 2 shows the output spectrum of the PM-HNLF for three different numbers of DWDM channels at the input. As seen in Fig. 2 (a) and (b), the idler is spectrally broadened due to the linear chirping by the pump pulse. Furthermore, different DWDM channels are mapped to different center wavelengths, since they all have the same temporal alignment with the pump pulse. Consequently, the bandwidth of the resulting OTDM signal is relatively broad, as seen in Fig. 2 (c). Mapping to the same central idler wavelength requires a time-delay between the DWDM channels
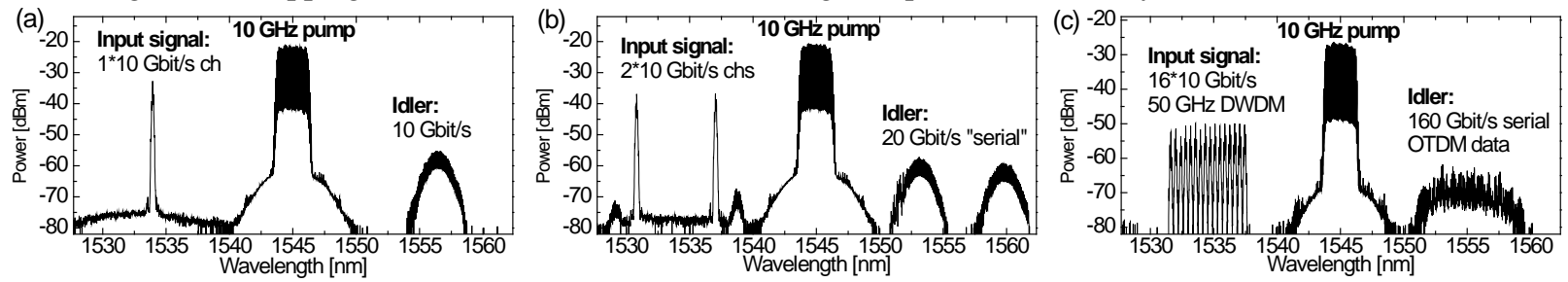

Fig. 2. Output spectra of the PM-HNLF for different DWDM inputs: (a) one channel, (b) two channels, and (c) 16 channels (res. $0.01 \mathrm{~nm}$ ). 

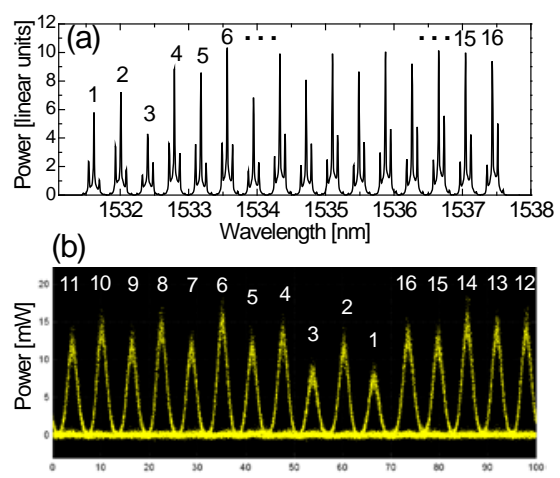

Time [ps] (span is $100 \mathrm{ps})$

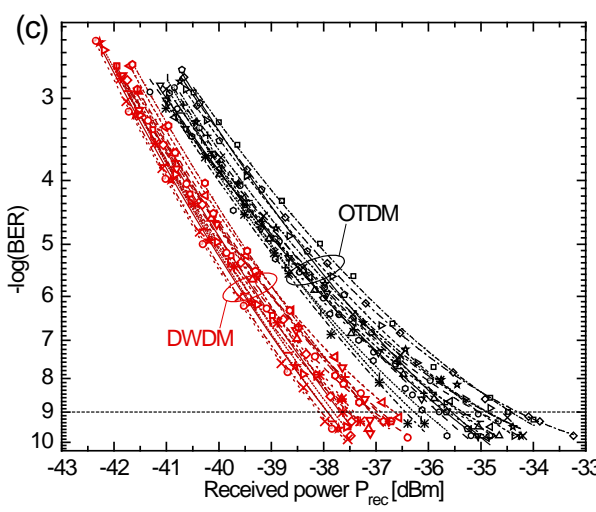

Fig. 3. Experimental results of $16 \times 10$ Gbit/s DWDM-OTDM conversion. (a) Spectrum of DWDM signal measured at the PM-HNLF output,

(b) Waveform of the output $160 \mathrm{Gbit} / \mathrm{s}$ OTDM signal (OSO trace), (c) BER curves of filtered DWDM channels and demultiplexed OTDM tributaries after conversion, (d) corresponding sensitivities and resulting penalty of the DWDM-OTDM conversion.

equal to the target OTDM bit slot, which could be achieved in this set-up by propagating the synchronous DWDM signal through an extra dispersive fibre before the PM-HNLF. However, it would imply that not all DWDM channels can be covered by the pump pulse and thereby converted to OTDM. The case of non-synchronous DWDM pulses will be examined in future work.

The 16×10 Gbit/s DWDM-OTDM conversion is successfully achieved, and the results are shown in Fig. 3. In Fig. 3 (a), a zoom-in on the DWDM spectrum from Fig. 2 (c) is shown. Fig. 3 (b) shows the temporal waveform of the OTDM signal obtained after the $875 \mathrm{~m}$ SMF. The 16 tributaries are periodically distributed over $100 \mathrm{ps}$, since a tributary spacing very close to the target $6.25 \mathrm{ps}$ is achieved. There is also a good correspondence with the DWDM spectrum in Fig. 3 (a), in terms of clearly resolved tributaries and relative powers. The variation in channel power is attributed to non-linear interactions in the PM-HNLF, and is not observable at the input. The DWDM temporal waveform displays strong intensity peaks, since all channels are coherent (see inset in Fig. 1). The resulting nonlinear effects in the PM-HNLF were minimised by lowering the average input power, but could not be completely avoided. For the BER measurement, each DWDM channel is extracted at the PM-HNLF output (pump signal off) by filtering with the OTF adjusted to a $\sim 0.3 \mathrm{~nm}$ bandwidth, and then sent into the receiver. Fig. 3 (c) shows the BER curves of the 16 DWDM channels, as well as the 16 OTDM tributaries after conversion and demultiplexing. All OTDM tributaries have error-free performance. The OTDM tributary sensitivities and the corresponding DWDM channel sensitivities at $\mathrm{BER}=10^{-9}$ are plotted in Fig. 3 (d). The relative penalties range from $1.1 \mathrm{~dB}$ to $3.2 \mathrm{~dB}$, with an average of $2.1 \mathrm{~dB}$. We expect an improved performance if the non-linear effects in the FWM medium attributed to the DWDM signal intensity peaks can be avoided, such as for a DWDM signal with non-coherent channels.

\section{Conclusion}

We have proposed DWDM-OTDM conversion by time-domain OFT, allowing simultaneous conversion of all channels using a single FWM-based switch. The scheme was successfully demonstrated by converting a synchronized 16×10 Gbit/s DWDM signal with $50 \mathrm{GHz}$ channel spacing to a $160 \mathrm{Gbit} / \mathrm{s}$ OTDM signal with $6.25 \mathrm{ps}$ tributary spacing. The BER performance was error-free with an average conversion penalty of only $2.1 \mathrm{~dB}$.

\section{Acknowledgement}

We would like to acknowledge OFS Fitel Denmark ApS for kindly providing the highly non-linear fibres, and the European Commission through the project EURO-FOS under the 7th Framework programme (ICT).

\section{References}

[1] G. Zarris, E. Hugues-Salas, N. A. Gonzalez, R. Weerasuriya, F. Parmigiani, D. Hillerkuss, P. Vorreau, M. Spyropoulou, S. K. Ibrahim, A. D. Ellis, R. Morais, P. Monteiro, P. Petropoulos, D. J. Richardson, I. Tomkos, J. Leuthold, and D. Simeonidou, "Field Experiments With a Grooming Switch for OTDM Meshed Networking,” J. Lightwave Technol. 28 (4), 316-327 (2010).

[2] X. Wu, A. Bogoni, S. Nuccio, O. F. Yilmaz, M. Scaffardi, and A. E. Willner, "High-Speed Optical WDM-to-TDM Conversion Using Fiber Nonlinearities,” IEEE J. Sel. Topics Quantum Electron. 16 (5), 1441-1447 (2010).

[3] M. Nakazawa, T. Hirooka, F. Futami, and S. Watanabe,"Ideal distortion-free transmission using optical Fourier transformation and Fourier transform-limited optical pulses”, IEEE Photon. Technol. Lett. 16 (4), 1059-1061 (2004).

[4] H. C. H. Mulvad, E. Palushani, M. Galili, J. Xu, H. Hu, A. Clausen, L. Oxenløwe, and P. Jeppesen, "OTDM-WDM conversion based on timedomain optical Fourier transformation with spectral compression”, Optical Fiber Communication Conference, OFC 2011, paper OThN2 (2011).

[5] R. Salem, M.A. Foster, A.C. Turner, D.F. Geraghty, M. Lipson and A.L. Gaeta, "Optical time lens based on four-wave mixing on a silicon chip”, Opt. Lett. 33 (10), 1047-1049 (2008). 